\title{
THE SUPPLY OF MAGNETIC FIELDS FROM A CD GALAXY
} TO INTRA-CLUSTER SPACE

\author{
H. HIRASHITA AND S. MINESHIGE \\ Department of Astronomy, Kyoto University, \\ Sakyo-ku, Kyoto 606-01, Japan \\ K. SHIBATA \\ National Astronomical Observatory, 2-21-1 Osawa, \\ Mitaka, Tokyo 181, Japan \\ AND \\ R. MATSUMOTO \\ Department of Physics, Faculty of Science, Chiba University, \\ Inage-ku, Chiba 263, Japan
}

\section{Introduction}

Intra-cluster spaces are filled with intra-cluster medium (ICM), whose typical temperature and density are $T_{\mathrm{ICM}} \sim 10^{7.5} \mathrm{~K}$ and $n_{\mathrm{ICM}} \sim 10^{-3} \mathrm{~cm}^{-3}$, respectively (e.g., Sarazin 1988). Recent Faraday rotation measurements have revealed the existence of magnetic fields in ICM with $B_{\mathrm{ICM}} \sim \mathrm{a}$ few $-10 \mu \mathrm{G}$ (e.g., Ge \& Owen 1993). In ICM, the plasma $\beta$ (the ratio of gas pressure to magnetic pressure) is almost "equipartition" value as follows:

$$
\beta \simeq 1 \times\left(\frac{B_{\mathrm{ICM}}}{10 \mu \mathrm{G}}\right)^{-2}\left(\frac{n_{\mathrm{ICM}}}{10^{-3} \mathrm{~cm}^{-3}}\right)\left(\frac{T_{\mathrm{ICM}}}{10^{7.5} \mathrm{~K}}\right) .
$$

However, the origin of the magnetic fields in ICM remains to be understood. We consider that fields may originate from central $\mathrm{cD}$ galaxies in clusters of galaxies. Here, we conjecture that the magnetic fields in $\mathrm{cD}$ galaxies can be lifted up to intra-cluster space by MHD instabilities. The most possible instability is the Parker instability (Parker 1966), which is a global MHD instability in a gravitational field. The growth time for the instability is estimated by the free-fall time. 


\section{Growth Rate of the Parker Instability}

\subsection{FIELD CONFIGURATION}

To estimate the growth time for the Parker instability in a cD galaxy, we examined linear stability of the magnetized medium in cylindrical coordinate $(r, \theta, z)$, whose origin is located at the center of the $c D$ galaxy. The gravitational field of the $\mathrm{cD}$ galaxy is radially inward and the magnetic field is assumed to be axially symmetric with curvature radius of $r$. Parker (1966) briefly discussed that the instability grows with the timescale of free-fall time in spite of the stabilizing effect of magnetic tension. Based on the formulation of Horiuchi et al. (1988), we confirmed Parker's result and derived the dispersion relation (Hirashita et al. 1998 in preparation).

\subsection{GROWTH RATE}

Using the dispersion relation we derived, we estimate typical growth time $\tau$ for the Parker instability in a cD galaxy:

$$
\tau \sim 10^{8}\left(\frac{H}{1 \mathrm{kpc}}\right)\left(\frac{v_{\mathrm{A}}}{100 \mathrm{~km} \mathrm{~s}^{-1}}\right)^{-1} \mathrm{yr},
$$

where $H$ and $v_{\mathrm{A}}$ are typical pressure scale height and Alfvén velocity in the cD galaxy, respectively. The growth time $\tau$ turns out to be shorter than a galaxy-evolution timescale $\left[\sim 10^{9}\right.$ yr (Binney \& Tremaine 1987)], which means that the Parker instability is an effective mechanism to supply magnetic fields to intra-cluster space.

\section{Conclusion}

Having examined properties of the Parker instability in a cD galaxy, we conclude that the Parker instability is an effective mechanism to supply magnetic fields to intra-cluster space.

The lifted-up magnetic fields may dissipate through magnetic reconnections, which may contribute effectively to the heating of ICM.

\section{References}

Binney, J. \& Tremaine, S. (1987) Galactic Dynamics, Princeton University Press, Princeton, p. 552

Ge, J. P. \& Owen, F. N. (1993) $A J$, 105, pp. 778-787

Horiuchi, T., Matsumoto, R., Hanawa, T., \& Shibata, K. (1988), PASJ, 40, pp. 147-169 Parker, E. N. (1966) ApJ, 145, pp. 811-833

Sarazin, C. L. (1988) X-Ray Emission from Clusters of Galaxies, Cambridge University Press, Cambridge 